\section{(2) OPEN ACCESS}

\title{
Chronobiological patterns of acute aortic dissection in central China
}

\author{
Liangtao Xia, ${ }^{1}$ Lu Huang, ${ }^{2}$ Xin Feng, ${ }^{1}$ Jiewen Xiao, ${ }^{1}$ Xiang Wei, ${ }^{1,3,4,5}$ Xinyu Yu (1) ${ }^{1}$
}

${ }^{1}$ Division of Cardiothoracic and Vascular Surgery, Tongji Hospital, Tongji Medical College, Huazhong University of Science and Technology, Wuhan, Hubei, China

${ }^{2}$ Tongji Hospital, Tongji Medical College, Huazhong University of Science and Technology, Wuhan, Hubei, China ${ }^{3}$ Key Laboratory of Organ Transplantation, Ministry of Education, Wuhan, Hubei, China ${ }^{4} \mathrm{NHC}$ Key Laboratory of Organ Transplantation, Wuhan, Hubei, China

${ }^{5}$ Key Laboratory of Organ Transplantation, Chinese Academy of Medical Sciences, Wuhan, Hubei, China

\section{Correspondence to} Dr Xinyu Yu and Prof Xiang Wei, Division of Cardiothoracic and Vascular Surgery, Tongji Hospital, Tongji Medical College Huazhong University of Science and Technology, Wuhan 430030, China; yuxinyu7@hust.edu.cn, xiangwei@tjh.tjmu.edu.cn

Received 31 March 2020 Revised 4 June 2020 Accepted 8 June 2020 Published Online First 13 July 2020

\begin{abstract}
Background Acute aortic dissection (AAD) is a lifethreatening emergency with poor clinical outcomes. Understanding the chronological patterns of $A A D$ onset would be helpful for identifying the triggers of $A A D$ and preventing this catastrophic event.

Methods We collected data from 2048 patients diagnosed with AAD at Tongji Hospital (Wuhan, China) from 2011 to 2018. The $\chi^{2}$ test was used to determine whether a specific period had significantly different seasonal/weekly distributions from other periods. Fourier models were used to analyse the rhythmicity in monthly/ circadian distribution.
\end{abstract}

Results The mean age was $53.4 \pm 10.9$ years, and 1161 patients (56.7\%) were under 55 years. One thousand six hundred fifty-seven patients (80.9\%) were male, and 935 cases (45.7\%) were type A dissections. The proportions of patients with comorbid hypertension/ diabetes were $60.3 \%$ (1234 cases) and 1.8\% (36 cases), respectively. A peak was identified in colder periods (winter/December) and a trough in warmer periods (summer/June). No significant variation was observed in weekly distribution. Fourier analysis showed a statistically significant circadian variation $(p<0.001)$ with a nocturnal trough in 2:00-3:00, a morning peak in 9:00-10:00, and an afternoon peak in 16:00-17:00. Subgroup analyses identified circadian rhythmicity in all subgroups except for the female group and younger group (younger than 55 years).

Conclusion Our results confirmed that the onset of AAD exhibits significant seasonal, monthly and circadian patterns. Patients with AAD with different Stanford-type dissections, sexes, ages and hypertension statuses could present different circadian variations. These findings may provide novel perspectives for identifying the triggers of $A A D$ and better preventing this catastrophic event.

\section{INTRODUCTION}

Acute aortic dissection (AAD) is a life-threatening emergency with high morbidity and mortality. ${ }^{1}$ The reported incidence of AAD is three to five cases per 100000 people every year worldwide, ${ }^{2}$ but this incidence might be underestimated since hospitalbased reports did not take preadmission deaths into account. A study based on a large cardiovascular registry indicated that more than $60 \%$ of $\mathrm{AAD}$ cases were not detected clinically but were first identified by autopsy. ${ }^{3}$ Therefore, despite the great advancements in therapeutic techniques, the early recognition and diagnosis of AAD are still of vital importance. Epidemiological evidence suggests that the onset of AAD occurs at different times with different probabilities. ${ }^{4}$ Analysing the chronobiological patterns of AAD could contribute to the prevention and timely treatment of this condition.

Currently, compelling evidence has revealed that myocardial infarction, stroke and arrhythmias occur more frequently in the morning hours, in winter and on Mondays. ${ }^{5-7}$ Disturbances to the internal circadian clock, modifications of behaviour patterns and fluctuations of environmental variables may contribute to the increased risks at specific times. However, limited studies have discussed how the time of day, week and year affect the onset of AAD, and most of these studies were conducted in Western countries with limited sample sizes and without subgroup analyses. ${ }^{8-13}$ To evaluate the effect of chronobiological patterns on the occurrence of $\mathrm{AAD}$, we aimed to expound the temporal trends of AAD onset in an 8-year timeseries study in central China, and we believe that these data could help predict and prevent the disastrous outcomes of this condition.

\section{METHODS}

\section{Study population and data collection}

This time-series study was reported according to the Strengthening the Reporting of Observational Studies in Epidemiology checklist. This study was based on electronic medical records of patients admitted to Tongji Hospital, Tongji Medical College, Huazhong University of Science and Technology, which is a major comprehensive hospital in central China that mainly treats patients from Hubei Province. We enrolled all patients diagnosed with AAD from 1 January 2011 to 31 December 2018 based on their electronic medical records. International Classification of Diseases-10 codes (I71.000-I71.005, I71.007) were used to preliminarily identify patients with aortic dissection (AD). Imaging information (ie, CT angiography and transoesophageal echocardiology) and surgical records of the included patients were checked for further confirmation. Since the clinical manifestations of AAD, intramural haematoma (IMH) and penetrating atherosclerotic ulcer (PAU) were similar and a clear diagnosis among the three was challenging, we included only patients who had imaging evidence or surgical records supporting the diagnosis of AAD. To analyse the time distribution of AAD onset, we set predefined exclusion criteria for the participants to improve the quality and validity of our research. The exclusion criteria were as follows: (1) AD without typical symptoms, defined as acute chest/back/abdominal pain within 14 days, ${ }^{2}$ since the onset time could not be
To cite: Xia L, Huang L, Feng $X$, et al. Heart 2021;107:320-325. 
accurately recalled; (2) simple IMH/simple PAU; (3) AD with congenital abnormalities, such as Marfan syndrome (MFS) or bicuspid aortic valve or a history of cardiovascular surgery/iatrogenic AD; (4) traumatic AD/AD in pregnancy; and (5) aortic aneurysm (since only dissections were considered for inclusion). The following baseline characteristics of the included patients were extracted separately: sex, age, Stanford type of AAD, and medical history of diabetes and hypertension. The onset date and exact time (if available) of AAD were inferred from the occurrence of acute symptoms based on electronic medical records.

\section{Study design}

We aimed to investigate four temporal patterns of AAD onset, including seasonal, monthly, weekly and circadian distributions. The included patients were categorised into 4 seasonal periods, 121 -month periods and 7 -day periods. Four seasons were defined according to the local climate conditions: spring from March to May, summer from June to August, autumn from September to November, and winter from December to February. For patients with a precise onset time, circadian rhythmicity was analysed after categorisation into 24 1-hour increments (eg, onset time between 0:00 and 0:59 categorised in the 0:00 group). Furthermore, we investigated whether differences in sex, age (younger than 55 years old, older or equal than 55 years old), AAD type (Stanford classification) and comorbid conditions such as hypertension would affect the chronobiology pattern, especially circadian variations.

\section{Statistical analysis}

Continuous variables following a normal distribution are described as the mean $\pm \mathrm{SD}$, while those that were non-normally distributed are described as the median (IQR). Categorical variables are described as percentages. The seasonal and weekly distributions of AAD onset were tested for uniformity among the total population and subgroups by the $\chi^{2}$ test. Values following uniform distribution were used as expected values to complete the test. For the monthly data, a Fourier model with one harmonic of 12 months was utilised to help analyse the trend and rhythmicity. For the circadian data, a Fourier model with four harmonics (periods of 24, 12, 8 and 6 hours) was chosen to fit the data based on the preliminary test. ${ }^{11} \mathrm{R}$ squared was used to represent the goodness of fit. The F statistic (hypothesis of

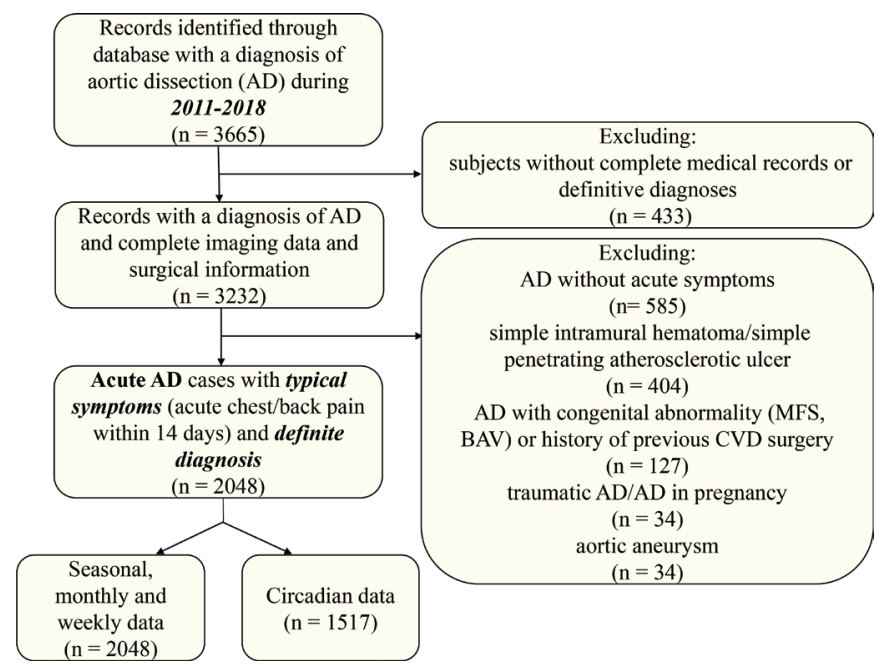

Figure 1 Flowchart of the study. BAV, bicuspid aortic valve; CVD, cardiovascular disease; MFS, Marfan syndrome.
Table 1 Characteristics of the study population

\begin{tabular}{llcc}
\hline Characteristics & $\begin{array}{l}\text { Overall } \\
(\mathbf{n}=2048)\end{array}$ & $\begin{array}{l}\text { Type A } \\
(\mathbf{n}=935)\end{array}$ & $\begin{array}{l}\text { Type B } \\
(\mathbf{n}=1113)\end{array}$ \\
\hline Age (years), mean (SD) & $53.4(10.9)$ & $52.7(10.8)$ & $54.0(11.0)$ \\
Male, $\mathrm{n}(\%)$ & $1657(80.9)$ & $728(77.9)$ & $929(83.5)$ \\
Hypertension, $\mathrm{n}(\%)$ & $1234(60.3)$ & $540(57.8)$ & $694(62.4)$ \\
Diabetes, $\mathrm{n}(\%)$ & $36(1.8)$ & $19(2.0)$ & $17(1.5)$ \\
\hline
\end{tabular}

straight-line model fitting) was reported to represent the statistical significance of the fitted function. A value of $p<0.05$ was considered significant in all tests. All statistical analyses were performed on GraphPad Prism V.8.3.0.

\section{Patient and public involvement statement}

This planned study was not presented to a patient group for their input prior to commencement of the study due to its retrospective nature. When we extracted data from the electronic medical records, we first deleted all personal information, including names and hospitalisation numbers, to ensure that the privacy of the patients would not be disclosed to the researchers and other involved members.

\section{RESULTS}

\section{Basic characteristics of the study population}

In total, 2048 patients with AAD with typical symptoms and definitive diagnoses were identified (see figure 1), including 935 Stanford type A dissections (45.7\%) and 1113 Stanford type $\mathrm{B}$ dissections (54.3\%). One thousand six hundred fifty-seven patients $(80.9 \%)$ were male. The average age was $53.4 \pm 10.9$ years, and 1161 patients $(56.7 \%)$ were younger than 55 years old. The proportions of patients comorbid with hypertension and diabetes were $60.3 \%$ and $1.8 \%$, respectively. Among the included patients, 1517 patients had a precise onset time of AAD in their medical records, and we included these patients in the circadian analysis. Details are shown in table 1.

\section{Seasonal, monthly and weekly variations}

The seasonal distribution of AAD showed significant differences $\left(\chi^{2}\right.$ 93.97, df $\left.3, p<0.0001\right)$ (see figure 2). In general, most cases occurred in winter $(34.2 \%, 701$ cases $)$, and the fewest occurred in summer $(14.8 \%, 303$ cases). This trend also existed in the group of Stanford type B dissections $\left(\chi^{2} 45.81\right.$, df $\left.3, p<0.0001\right)$, with 399 cases $(35.8 \%)$ occurring in winter and 176 cases $(15.8 \%)$ occurring in summer. For Stanford type A dissections $\left(\chi^{2}\right.$ 55.12, df 3, p<0.0001), the fewest cases also occurred

\section{Seasonal distributions of AAD onset}

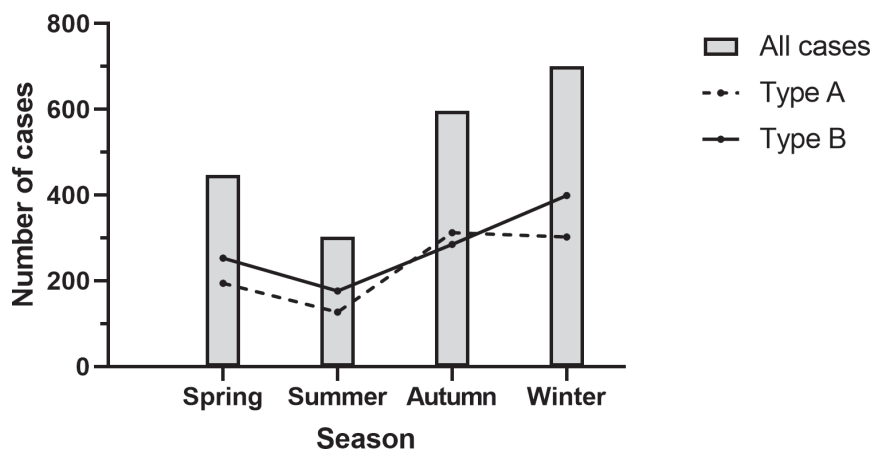

Figure 2 Seasonal distributions of $A A D$ onset. $A A D$, acute aortic dissection. 


\section{Monthly variation of AAD onset All cases}

$\left(n=2048 r^{2}=0.9118 p<0.0001\right)$

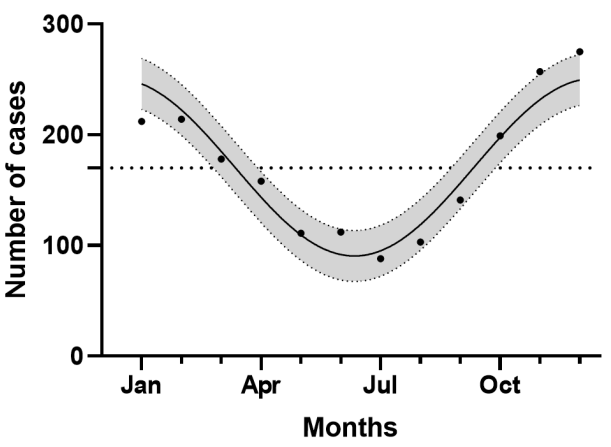

Monthly variation of AAD onset Type A cases $\left(n=935 r^{2}=0.8611 p<0.0001\right)$

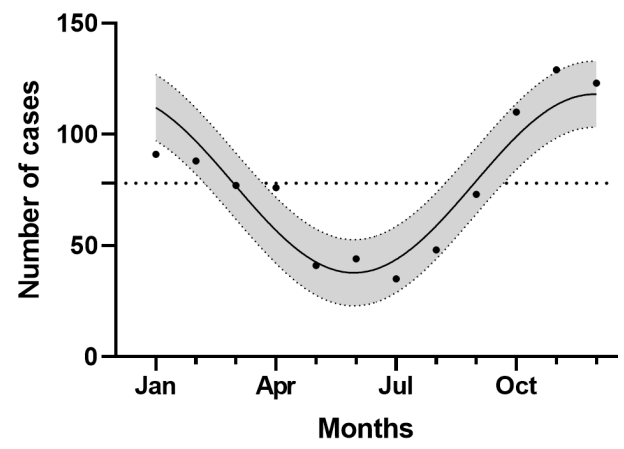

Monthly variation of $A A D$ onset Type B cases $\left(n=1113 r^{2}=0.8994 p<0.0001\right)$

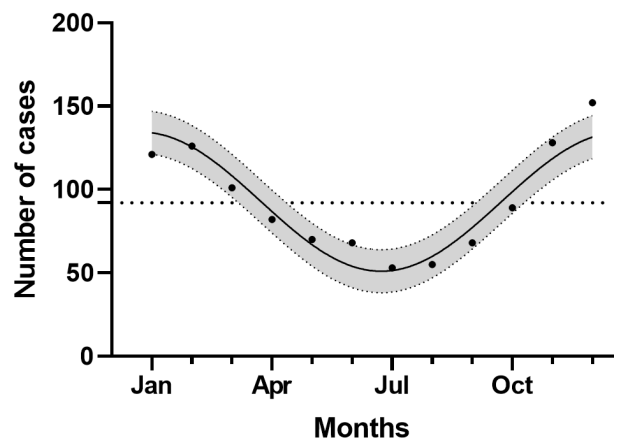

Figure 3 Circannual variations of AAD onset for all cases, type $A$ cases and type $B$ cases, using a Fourier model. Each point represents the total count of onset AAD for the periods considered. The active line is overall best-fitting curve calculated by rhythm analysis, resulting from one significant harmonic of 12 months. The filled area represents $95 \%$ $\mathrm{Cl}$. The dotted line represents the midline estimated statistic of rhythm. $A A D$, acute aortic dissection.

in summer $(13.7 \%, 127$ cases), and the number of cases that occurred in autumn $(33.4 \%, 312$ cases) was almost equal to that in winter $(32.3 \%, 302$ cases).

Fourier rhythm analysis identified a statistically significant temporal pattern $(\mathrm{p}<0.001)$ in monthly distribution (see figure 3). A peak was observed around December, and a nadir
Weekly distributions of AAD onset

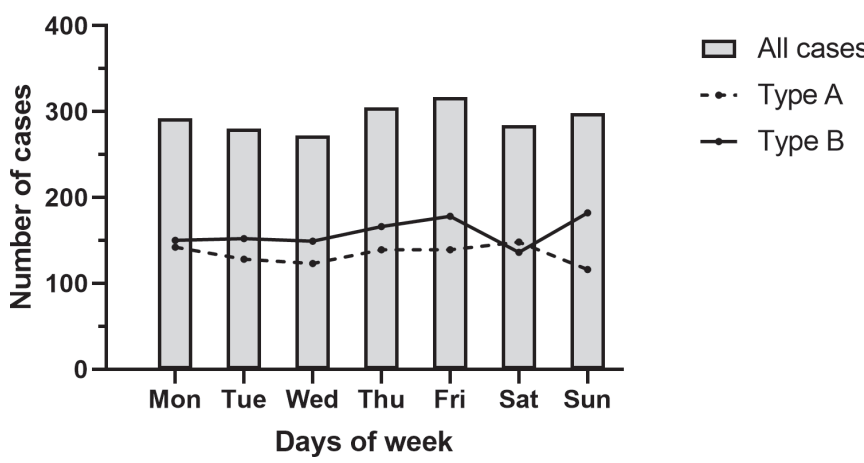

Figure 4 Weekly distributions of $A A D$ onset. $A A D$, acute aortic dissection.

was observed around June. Similar patterns were detected in patients with type $\mathrm{A}$ and type $\mathrm{B}$ dissections.

The weekly distribution of AAD is displayed in figure 4. No statistically significant difference was observed in the number of cases on different days of the week $\left(\chi^{2} 2.439\right.$, df $\left.6, p=0.875\right)$, neither in patients with type A dissection $\left(\chi^{2} 2.998\right.$, df 6, $\mathrm{p}=0.809)$ nor in patients with type B dissection $\left(\chi^{2} 5.311\right.$, df $6, \mathrm{p}=0.505)$.

\section{Circadian variations}

Fourier rhythm analysis identified statistically significant circadian patterns among the total population $(p<0.001)$, with a nocturnal trough between 2:00 and 3:00, a minor morning peak between 9:00 and 10:00, and a major afternoon peak between 16:00 and 17:00 (see figure 5).

Similar trends were detected in all subgroups except for the female group $(p=0.164)$ and the younger group $(p=0.080)$. The nocturnal trough appeared between 2:00 and 4:00 in all groups

\section{Circadian variation of AAD onset All cases $\left(n=1517 r^{2}=0.8151 p=0.0008\right)$}

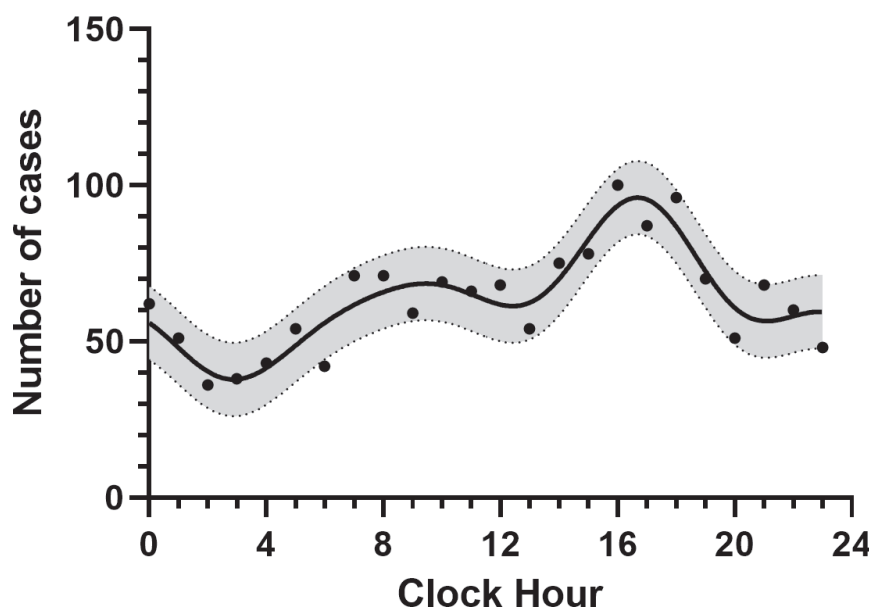

Figure 5 Circadian variation of AAD onset for all cases using a Fourier model. Each point represents the total count of onset AAD for the periods considered. The active line is the overall best-fitting curve calculated by rhythm analysis, resulting from four significant harmonics with periods of $24,12,8$ and 6 hours. The filled area represents $95 \% \mathrm{Cl}$. $A A D$, acute aortic dissection. 
Table 2 Circadian variations of hourly data of AAD onsets, fitted by Fourier model

\begin{tabular}{|c|c|c|c|c|c|c|c|c|c|}
\hline Group & $\mathrm{N}$ & Nocturnal trough & $\mathrm{n}$ & Morning peak & $\mathrm{n}$ & Afternoon peak & $\mathrm{n}$ & $\mathrm{R}$ squared & $P$ value \\
\hline Total & 1517 & $2: 52$ & $37.824 \pm 11.747$ & 9:27 & $68.506 \pm 11.747$ & $16: 41$ & $96.015 \pm 11.747$ & 0.8151 & 0.0008 \\
\hline Type A & 737 & $2: 36$ & $15.432 \pm 7.618$ & $8: 29$ & $37.323 \pm 7.618$ & $16: 41$ & $44.097 \pm 7.618$ & 0.7203 & 0.0052 \\
\hline Type B & 780 & 4:03 & $21.304 \pm 8.914$ & $10: 00$ & $31.192 \pm 8.914$ & $16: 41$ & $51.917 \pm 8.914$ & 0.7204 & 0.0143 \\
\hline Male & 1242 & $2: 50$ & $29.235 \pm 9.248$ & 9:53 & $56.591 \pm 9.248$ & $16: 39$ & $79.225 \pm 9.248$ & 0.826 & 0.0004 \\
\hline Female & 275 & $4: 58$ & $7.063 \pm 5.282$ & $8: 46$ & $12.500 \pm 5.282$ & $18: 29$ & $17.290 \pm 5.282$ & 0.5518 & 0.1641 \\
\hline Age $<55$ years & 875 & 4:07 & $23.327 \pm 10.628$ & $8: 54$ & $40.316 \pm 10.628$ & $16: 51$ & $52.087 \pm 10.628$ & 0.5849 & 0.0803 \\
\hline Age $\geq 55$ years & 642 & $2: 18$ & $11.387 \pm 4.919$ & $10: 40$ & $29.974 \pm 4.919$ & $16: 34$ & $44.026 \pm 4.919$ & 0.8766 & $<0.0001$ \\
\hline Hypertensive & 929 & 3:02 & $23.861 \pm 9.927$ & $7: 00$ & $36.635 \pm 9.927$ & $16: 50$ & $61.886 \pm 9.927$ & 0.7238 & 0.0088 \\
\hline Normotensive & 577 & $1: 57$ & $13.552 \pm 4.843$ & 9:32 & $34.235 \pm 4.843$ & $16: 14$ & $33.659 \pm 4.843$ & 0.8375 & 0.0002 \\
\hline
\end{tabular}

Letter $\mathrm{N}$ indicates the number of cases in each group; letter $\mathrm{n}$ shows the number of cases fitted by Fourier model, presented by numbers $\pm 95 \%$ Cls; $\mathrm{R}$ square represents goodness of the model; and $\mathrm{P}$, probability value resulting from the $\mathrm{F}$ statistic used to test hypothesis of straight-line model fitting.

$A A D$, acute aortic dissection.

except in the female group (at 4:58). Most subgroups showed a morning peak between 8:00 and 10:00, except for an earlier peak for the hypertensive group (at 7:00) and a delayed peak for the older group (at 10:40). The afternoon peak occurred between 16:00 and 17:00 in all subgroups except the female group, which had a peak at approximately 18:29. Detailed information is displayed in table 2 and figure 6.

\section{DISCUSSION}

We conducted a single-centre retrospective study including 2048 patients from central China to identify potential chronobiological patterns of AAD onset. First, we recognised a higher incidence in the colder period, such as in winter or December, and a lower incidence in the warmer period, such as in summer or June. These results are consistent with most previous studies, ${ }^{8} 1114$ and the winter peak was reported to be independent of climate conditions. $^{15}$ Lower temperature is associated with increased sympathetic activity and blood pressure (BP), as well as changes in haematological properties (including increased blood viscosity, haemoglobin, mean corpuscular volume, platelet aggregability, factor VII, fibrinogen, and acute-phase reactants and decreased red cell deformability). ${ }^{4}{ }^{8}$ Moreover, the concentrations of air pollutants, especially fine particulate matter, are also elevated in winter and are associated with aortic plaque progression, hypertension and aortic constriction. ${ }^{9}$ These changes may lead to a surge in the shear forces exerted on the aortic intima, triggering AAD in colder months. ${ }^{8}$

Second, no significant weekly variation was observed for AAD onset in our study. The results from previous studies on the weekly distribution of AAD onset remain conflicting. Some studies reported a Monday peak, ${ }^{12} 1416$ while others reported a Wednesday peak. ${ }^{9}$ The weekly distribution of cardiovascular disease (CVD) may reflect physiological fluctuations resulting from an increase in physical and mental burdens from leisurely weekend activities to stressful weekdays. ${ }^{17}$ Different working statuses and occasional holidays may easily alter the weekly patterns of AAD onset, leading to a non-significant result.

Moreover, we demonstrated a significant circadian pattern in AAD onset. For the entire studied population, a nocturnal trough appeared at 2:52, followed by a minor morning peak at 9:27 and a major afternoon peak at 16:41. Indeed, a similar daily pattern has been found in the occurrence of many adverse cardiovascular events, including stroke, myocardial infarction and sudden cardiac death, which all occur most frequently in the morning. ${ }^{7}$ Such a circadian pattern may result from fluctuations in BP. It has been known for several decades that BP exhibits a circadian rhythm in humans due to an innate circadian rhythm,
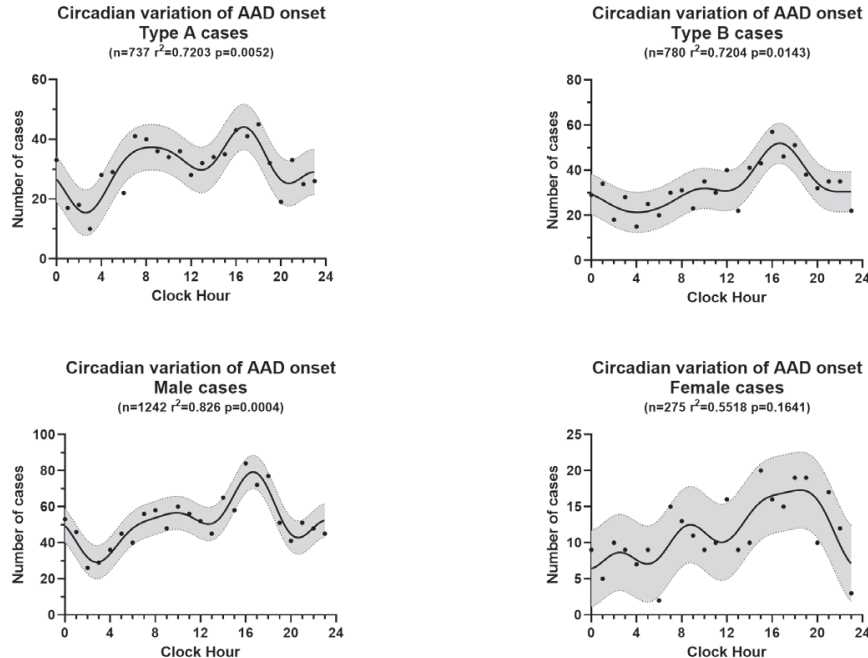

Circadian variation of AAD onset Female cases
$(n=275$
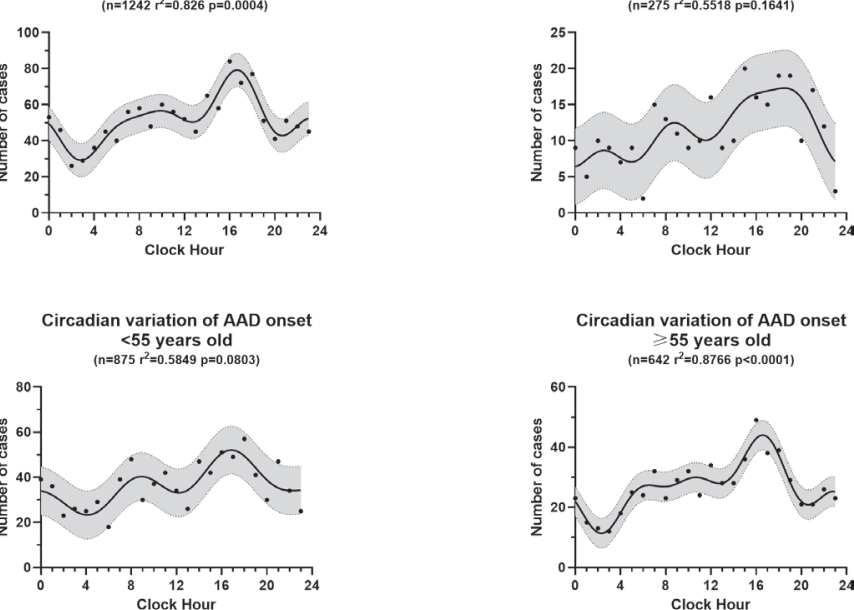

Circadian variation of AAD onset with Hypertension

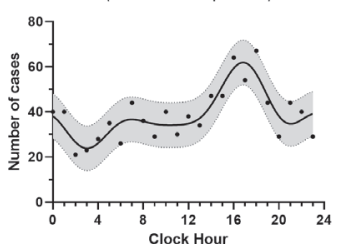

Circadian variation of $A A D$ onset without Hypertension

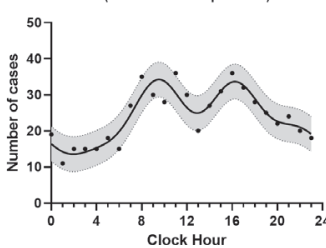

Figure 6 Circadian variations of AAD onset for different subgroups using a Fourier model. Each point represents the total count of onset AAD for the periods considered. The active line is the overall best-fitting curve calculated by rhythm analysis, resulting from four significant harmonics with periods of 24, 12, 8 and 6 hours. The filled area represents $95 \% \mathrm{Cl}$. AAD, acute aortic dissection.

as well as external stimulation from environmental fluctuations and daily behaviours-BP dips at night during rest, undergoes a steep increase in the morning (known as the 'morning surge') 
and peaks typically in the late afternoon. ${ }^{18}$ Moreover, the reactivity of BP to daily physical activity has been reported to show 24-hour variations, in which systolic BP reactivity was highest in the morning and exhibited a secondary rise in the afternoon. ${ }^{19}$ Most cardiovascular-related variables exhibit a circadian rhythm with a morning peak, including heart rate, operation of the renin-angiotensin-aldosterone system, vascular resistance and sympathetic activity. ${ }^{7}$ In addition, the balance between coagulation and fibrinolysis is also disturbed, resulting in hypercoagulability and hypofibrinolysis in the morning. ${ }^{20}$ The surges in BP and BP reactivity, together with other changes in the cardiovascular system, would increase arterial shear force, triggering the onset of AAD in corresponding periods, especially in people with a genetic predisposition.

To further investigate the temporal pattern, we performed circadian analyses in subpopulations and identified some intriguing nuances. Patients with type A dissection showed a higher morning surge compared with patients with type B dissection. Type A dissection is closely related to inherited connective tissue diseases, while type B dissection is more likely to be related to atherosclerosis. ${ }^{21}$ However, previous studies on a genetic susceptible model (patients with MFS) ${ }^{22}$ and an atherosclerotic model (patients with spontaneous rupture of abdominal aortic aneurysm) $)^{23}$ reported similar circadian patterns. Whether patients with different types of AAD present different circadian patterns needs further investigation. Moreover, female patients did not exhibit a significant circadian pattern. Women with AAD have different clinical features and were reported to have a temporal delay from symptom onset to diagnosis, which may partly explain the non-significant result. ${ }^{1}$ The smaller sample size can also affect the model fitting process, leading to the non-significant result. In addition, the normotensive group exhibited a more obvious morning peak than the hypertensive group. Notably, abnormalities or loss of circadian rhythmicity in BP is common in certain forms of arterial hypertension, which may change the original onset patterns of AAD. ${ }^{24}$ Treatment for hypertension may also alter the innate circadian pattern of BP. However, whether treatment for hypertension or hypertension itself leads to the atypical morning peak needs further investigation. We also found that the younger patients exhibited a later nocturnal trough (at 4:07) than the older patients (at 2:18), which might be partially due to the different sleeping and waking habits between young and old patients.

A number of epidemiological studies have explored circadian patterns of AAD onset. In 2002, Mehta et al ${ }^{11}$ conducted a study that included 957 patients with AAD from around the world enrolled in the International Registry of Aortic Dissection and used non-linear Fourier analysis for the first time. They reported a nocturnal trough, a major early morning peak and a minor afternoon peak. Vitale et al ${ }^{14}$ performed a meta-analysis of eight studies, analysing hourly variations using the data from 1816 patients. They reported an increased risk of acute aortic rupture or dissection between 6:00 and 12:00 compared with the risk in other hours. Takagi et $a l^{25}$ analysed data from seven studies with 1827 patients with $\mathrm{AAD}$, and they identified a peak in 8:0010:00 and a nadir in 0:00-2:00. In this study, we identified a higher afternoon peak among Chinese patients with $\mathrm{AAD}$, and some Asian studies reported similar results. ${ }^{13} 2627$ This nuance could result from different social and dietary habits in different cultures. Asians tend to consume strong liquor and salty food at dinner in the late afternoon, which might account for the elevation in $\mathrm{BP}$ and contributes to the higher afternoon peak of AAD onset. ${ }^{132}$ We expect more studies on various ethnicities to elucidate this difference.
In summary, we thoroughly explored the seasonal, monthly, weekly and circadian patterns of AAD onset in an 8-year timeseries study in central China. Understanding the chronological patterns of AAD onset could be helpful for preventing this catastrophic event. In addition, we performed circadian analyses among subgroups and identified some intriguing nuances. These findings may provide novel perspectives for clinicians to understand the triggers of AAD. Moreover, with the identified circadian patterns of AAD onset, we should place more emphasis on chronotherapy for AAD. The intake of antihypertension or anticoagulation drugs in the evening could better match the daily physiological oscillations and significantly reduce CVD morbidity and mortality. ${ }^{29} 30$ These factors could probably benefit people with risk factors for $\mathrm{AD}$, such as hypertension or atherosclerosis.

There are several limitations in this study. First, our study had a single-centre retrospective design despite the large study population. There could be inclusion bias from using data extracted from electronic medical records, and part of the data were based on patient recall. Second, given that AAD has a high mortality rate within hours of onset, patients might fail to reach a hospital for timely medical treatment. Failure to include these patients inevitably affects the accuracy of the results.

\section{CONCLUSION}

In conclusion, we used data from 2048 Chinese patients and confirmed that the onset of AAD exhibits significant circannual and circadian patterns. For circannual variations, a peak was identified in colder periods, and a trough was identified in warmer periods. For daily variations, we identified a nocturnal trough, a morning peak and an afternoon peak. Differences in AD type, sex, age and hypertension status could lead to different circadian variations. These findings may provide novel perspectives to help us identify the triggers of AAD and better prevent this catastrophic event.

\section{Key messages}

What is already known on this subject?

- Epidemiological evidence suggests that the onset of AAD exhibits significant circadian and seasonal/monthly variations. However, most of these studies were conducted in Western countries and lack subgroup analyses.

\section{What might this study add?}

- This 8-year time-series study used data from 2048 patients in central China and identified significant seasonal, monthly and circadian patterns of AAD onset. More AAD cases occurred in colder periods (winter/December), and fewer cases occurred in warmer periods (summer/June). Rhythm analysis indicated a nocturnal trough, a morning surge and an afternoon peak in circadian patterns. Patients with different AAD types, sexes, ages and hypertension statuses presented different circadian variations.

How might this impact on clinical practice?

- Understanding the chronological patterns of AAD onset will help clinicians identify the triggers of $A A D$ and contribute to the prevention and timely treatment of this condition.

Acknowledgements Thanks are due to Professor Shiyi Cao, Dr Zhiyuan Li and Dr Penghui Yuan for assistance with the statistical analyses. 
Contributors $L X, X Y$ and $X W$ conceived the idea. Data collection and analysis were carried out by $L X$ and $X Y$. LX, XF and JX contributed to the interpretation of the results. $L X$ and $L H$ took the lead in writing the manuscript. $X W$ and $X Y$ are guarantors. All authors provided critical feedback and helped shape the research, analysis and manuscript.

Funding This study was supported by the National Natural Science Foundation of China (81670050).

Competing interests None declared.

Patient and public involvement Patients and/or the public were involved in the design, conduct, reporting or dissemination plans of this research. Refer to the Methods section for further details.

Patient consent for publication Not required.

Ethics approval This study was approved by the Research Ethics Commission of Tongji Medical College (2019-S1149). Informed consent form was not applicable for this study.

Provenance and peer review Not commissioned; externally peer reviewed.

Data availability statement All data relevant to the study are included in the article or uploaded as supplementary information.

Open access This is an open access article distributed in accordance with the Creative Commons Attribution Non Commercial (CC BY-NC 4.0) license, which permits others to distribute, remix, adapt, build upon this work non-commercially, and license their derivative works on different terms, provided the original work is properly cited, appropriate credit is given, any changes made indicated, and the use is non-commercial. See: http://creativecommons.org/licenses/by-nc/4.0/.

ORCID iD

Xinyu Yu http://orcid.org/0000-0002-3689-980X

\section{REFERENCES}

1 Evangelista A, Isselbacher EM, Bossone E, et al. Insights from the International registry of acute aortic dissection: a 20-year experience of collaborative clinical research. Circulation 2018;137:1846-60.

2 Nienaber CA, Clough RE, Sakalihasan N, et al. Aortic dissection. Nat Rev Dis Primers 2016;2:16053.

3 Huynh $\mathrm{N}$, Thordsen S, Thomas T, et al. Clinical and pathologic findings of aortic dissection at autopsy: review of 336 cases over nearly 6 decades. Am Heart J 2019:209:108-15.

4 Manfredini R, Fabbian F, Manfredini F, et al. Chronobiology in aortic diseases - "is this really a random phenomenon?". Prog Cardiovasc Dis 2013:56:116-24.

5 Gallerani M, Pala M, Fedeli U. Circaseptan periodicity of cardiovascular diseases. Heart Fail Clin 2017:13:703-17.

6 Stewart S, Keates AK, Redfern A, et al. Seasonal variations in cardiovascular disease. Nat Rev Cardiol 2017;14:654-64.

7 Thosar SS, Butler MP, Shea SA. Role of the circadian system in cardiovascular disease. J Clin Invest 2018;128:2157-67.

8 Kumar N, Pandey A, Venkatraman A, et al. Seasonality in acute aortic dissection related hospitalizations and mortality in the United States: a nationwide analysis from 2004-2011. Int J Cardiol 2015;179:321-2.
9 Ma W-G, Li B, Zhang W, et al. Chronologic and climatic factors of acute aortic dissection: study of 1642 patients in two continents. Ann Thorac Surg 2019. doi:10.1016/j.athoracsur.2019.11.013. [Epub ahead of print: 28 Dec 2019].

10 Manfredini R, Boari B, Gallerani M, et al. Chronobiology of rupture and dissection of aortic aneurysms. J Vasc Surg 2004;40:382-8.

11 Mehta RH, Manfredini R, Hassan F, et al. Chronobiological patterns of acute aortic dissection. Circulation 2002;106:1110-5.

12 Sumiyoshi M, Kojima S, Arima M, et al. Circadian, Weekly, and seasonal variation at the onset of acute aortic dissection. Am J Cardiol 2002;89:619-23.

13 Zhang L, Qiu J, Yang X, et al. Circadian variations in the onset of aortic dissection in northern China. Chronobiol Int 2018;35:1481-9.

14 Vitale J, Manfredini R, Gallerani M, et al. Chronobiology of acute aortic rupture or dissection: a systematic review and a meta-analysis of the literature. Chronobiol Int 2015;32:385-94.

15 Mehta $\mathrm{RH}$, Manfredini $\mathrm{R}$, Bossone $\mathrm{E}$, et al. The winter peak in the occurrence of acute aortic dissection is independent of climate. Chronobiol Int 2005;22:723-9.

16 Gallerani M, Imberti D, Bossone $\mathrm{E}$, et al. Higher mortality in patients hospitalized for acute aortic rupture or dissection during weekends. J Vasc Surg 2012;55:1247-54.

17 Willich SN, Löwel H, Lewis M, et al. Weekly variation of acute myocardial infarction. increased Monday risk in the working population. Circulation 1994;90:87-93.

18 Douma LG, Gumz ML. Circadian clock-mediated regulation of blood pressure. Free Radic Biol Med 2018;119:108-14.

19 Jones $\mathrm{H}$, Atkinson $\mathrm{G}$, Leary $\mathrm{A}$, et al. Reactivity of ambulatory blood pressure to physical activity varies with time of day. Hypertension 2006;47:778-84.

20 Budkowska M, Lebiecka A, Marcinowska Z, et al. The circadian rhythm of selected parameters of the hemostasis system in healthy people. Thromb Res 2019;182:79-88.

21 Cifani N, Proietta M, Tritapepe L, et al. Stanford-A acute aortic dissection, inflammation, and metalloproteinases: a review. Ann Med 2015:47:441-6.

22 Siddiqi HK, Luminais SN, Montgomery D, et al. Chronobiology of acute aortic dissection in the Marfan syndrome (from the National Registry of genetically triggered thoracic aortic aneurysms and cardiovascular conditions and the International registry of acute aortic dissection). Am J Cardio/ 2017:119:785-9.

23 Manfredini R, Portaluppi F, Zamboni P, et al. Circadian variation in spontaneous rupture of abdominal aorta. Lancet 1999:353:643-4.

24 Smolensky MH, Hermida RC, Portaluppi F. Circadian mechanisms of 24-hour blood pressure regulation and patterning. Sleep Med Rev 2017;33:4-16.

25 Takagi $\mathrm{H}$, Ando T, Umemoto T, et al. Meta-Analysis of circadian variation in the onset of acute aortic dissection. Am J Cardiol 2017;120:1662-6.

26 Kojima S, Sumiyoshi M, Nakata Y, et al. Triggers and circadian distribution of the onset of acute aortic dissection. Circ J 2002;66:232-5.

27 Ryu HM, Lee JH, Kwon YS, et al. Examining the relationship between triggering activities and the circadian distribution of acute aortic dissection. Korean Circ $J$ 2010:40:565-72

28 Sogunuru GP, Kario K, Shin J, et al. Morning surge in blood pressure and blood pressure variability in Asia: evidence and statement from the hope Asia network. J Clin Hypertens 2019;21:324-34.

29 Brunner-Ziegler S, Jilma B, Schörgenhofer C, et al. Comparison between the impact of morning and evening doses of rivaroxaban on the circadian endogenous coagulation rhythm in healthy subjects. J Thromb Haemost 2016;14:316-23.

30 Hermida RC, Crespo JJ, Domínguez-Sardiña M, et al. Bedtime hypertension treatment improves cardiovascular risk reduction: the Hygia chronotherapy trial. Eur Heart J 2019. doi:10.1093/eurheartj/ehz754. [Epub ahead of print: 22 Oct 2019]. 